\title{
The estrogen-like protective effect of Lycium barbarum polysaccharides in reducing oxidative stress on myocardial cells from ovariectomized rats
}

\author{
NING YU ${ }^{1}$, NAN SONG ${ }^{2}$, CHUN YING LIU $^{1}$ and GUAN LIN YANG ${ }^{2}$ \\ ${ }^{1}$ Graduate School; ${ }^{2}$ Key Laboratory of Ministry of Education for TCM Viscera-State Theory and Applications; \\ Liaoning University of Traditional Chinese Medicine, Shenyang, Liaoning 110847, P.R. China
}

Received September 27, 2016; Accepted November 10, 2017

DOI: $10.3892 / \mathrm{mmr} .2019 .9880$

\begin{abstract}
Previous studies have demonstrated that ovariectomy may lead to a reduction in antioxidative biomarkers in the myocardium, thus suggesting that estrogens may serve a protective role in the suppression of oxidative stress. Lycium barbarum polysaccharides (LBP) are a well-known antioxidant Chinese traditional medicine, which appear to have a similar function to estrogens with regards to the regulation of cardiac function. In the present study, 30 Sprague-Dawley rats were randomly divided into the following groups: Sham operation group, ovariectomized (OVX) group, estradiol valerate group, high-dose LBP (LBP-H) group and low-dose LBP (LBP-L) group. All of the rats were provided tap water, estradiol valerate or LBP for 12 weeks. In addition, all rats were ovariectomized, with the exception of rats in the sham operation group, which underwent fat removal only. Reactive oxygen species (ROS), malondialdehyde (MDA), glutathione peroxidase (GSH-px), catalase (CAT) and superoxide dismutase activities were subsequently examined. The protein expression levels of cleaved caspase-9, cleaved caspase-3 and phosphorylated-protein kinase B (p-Akt) were also assessed. The results demonstrated that high-dose LBP decreased the enhanced levels of ROS and MDA in OVX rats, whereas GSH-px and CAT activities were increased in the LBP-H group compared with in OVX rats. Furthermore, the expression levels of cleaved caspase- 9 and cleaved caspase-3 were significantly upregulated in the OVX group, whereas high-dose LBP exerted protective effects on OVX rats by decreasing the expression of apoptotic proteins. Conversely,
\end{abstract}

Correspondence to: Professor Guan Lin Yang, Key Laboratory of Ministry of Education for TCM Viscera-State Theory and Applications, Liaoning University of Traditional Chinese Medicine, 79 Chongshan Easten Road, Huanggu, Shenyang, Liaoning 110847, P.R. China

E-mail: zyzxsys@163.com

Key words: ovariectomy, oxidative stress, Lycium barbarum polysaccharides, apoptosis
p-Akt expression was decreased in the OVX group and was increased in the LBP-H group. These results indicated that LBP is essentially involved in cardiac protection by inhibiting apoptosis in response to oxidative stress. In addition, improvement of antioxidant status by LBP is associated with the Akt signaling pathway in the myocardium of OVX rats.

\section{Introduction}

Cardiovascular diseases are associated with a high rate of mortality in humans. In addition, cardiovascular diseases are less prevalent in women aged between 20 and 50 years compared with the corresponding male population. However, in individuals $>50$ years old, the incidence of cardiovascular disease is equivalent in both sexes $(1,2)$. Previous studies have reported that the presence of estrogens serves an important role in protection against cardiac injury, thus suggesting that menopause may be a risk factor for numerous cardiovascular diseases $(3,4)$. Studies in animal models have demonstrated that a lack of ovarian hormones, in particular estrogens, has detrimental effects on various organs, including the cardiovascular system $(5,6)$. Hormone replacement therapy (HRT) has therefore been recommended to postmenopausal women; however, the controversies regarding the safety of HRT have drawn attention to novel therapies for postmenopausal women (3,7-9).

Lycium barbarum polysaccharides (LBP) may be used in TCM to prevent postmenopausal symptoms. Clinical research has indicated that polysaccharides extracted from Lycium barbarum may serve an important biological role. LBP is composed of arabinose, glucose, galactose, mannose, xylose and rhamnose monosaccharide, etc., and contains various trace elements and amino acids (10). A previous study demonstrated that LBP is the main active ingredient of the TCM medlar, which can regulate immunity and improve age-associated symptoms, including fatigue, loss of appetite and blurred vision, and may reduce blood lipid levels and fatty liver disease, and exert anti-aging effects (11).

Oxidative stress reflects an imbalance between the systemic manifestation of reactive oxygen species (ROS) and the ability of the body to readily detoxify reactive intermediates or to repair the resulting damage (12). Numerous studies 
have demonstrated that oxidative stress is an important factor underlying abnormal cardiovascular system structure (13-15). In addition, various cardiovascular diseases, including hypertension, atherosclerosis, myocardial ischemia, ischemia-reperfusion injury, myocardial hypertrophy and heart failure, are associated with an increase in ROS generation. Furthermore, a previous study revealed that estrogen exerts antioxidative effects, which may serve a role in cardiac protection (16).

Increased cardiovascular risk in postmenopausal women may be due to the postmenopausal reduction in estrogen levels; therefore, the antioxidative effects are weakened and cardiovascular disease may be initiated. In the present study, LBP exerted a protective effect against heart failure in rats. In addition, LBP has been reported to reduce isopropyl adrenaline-induced heart failure and rat heart mass/weight ratio, reduce myocardial injury and significantly improve cardiac function in rats; the underlying mechanism may be associated with an improvement in antioxidant enzyme activity and a reduction in lipid peroxide formation $(17,18)$. The present study investigated whether LBP effects the oxidative stress state and induces antioxidative effects in the myocardium of ovariectomized (OVX) rats. In addition, the expression levels of apoptotic proteins and Akt pathway proteins were also detected in the myocardium. The present study aimed to explore whether LBP exerts protective effects against oxidative insult in OVX rats.

\section{Materials and methods}

Animals. A total of 30 female Sprague-Dawley rats (aged 10-12 weeks, weight 200 \pm 10 g) were purchased from Vital River Laboratories Co., Ltd., (Beijing, China). The rats were acclimated for 7 days, prior to use in subsequent experiments, and were housed in specific pathogen-free conditions (temperature $22 \pm 1^{\circ} \mathrm{C}$, humidity $50 \pm 5 \%$ ) under a 12 -h light/dark cycle. Tap water and chow were provided ad libitum. All efforts were made to minimize suffering, and procedures were performed under chloral hydrate $(300 \mathrm{mg} / \mathrm{kg}$, i.p.) anesthesia when necessary. The present study was approved by and followed the guidelines of the Animal Ethics Committee of Liaoning University of Traditional Chinese Medicine (Shenyang, China; permit no. 2011-167).

Experimental protocol. The adult female Sprague-Dawley rats were randomly divided into the following five groups ( $n=6 /$ group): i) Sham operation group, in which a small region of fat was removed via bilateral paraspinal incisions, and rats were fed with tap water for 12 weeks; ii) OVX group, in which the ovaries of the rats were exteriorized and removed via bilateral paraspinal incisions, and rats were fed tap water for 12 weeks; ii) estradiol valerate group (Est), in which rats were fed estradiol valerate $(0.105 \mathrm{mg} / \mathrm{kg})$ for 12 weeks following OVX; iv) high-dose LBP group (LBP-H), in which rats were fed LBP (250 mg/kg) for 12 weeks following OVX; v) low-dose LBP group (LBP-L), in which rats were fed LBP $(125 \mathrm{mg} / \mathrm{kg}$ ) following OVX. All procedures were performed under $10 \%$ chloral hydrate anesthesia $(300 \mathrm{mg} / \mathrm{kg}$ ). Following surgery, all rats received prophylactic antibiotic therapy (penicillin G procaine; 4,000 IU/kg i.m.). Daily vaginal smears were collected from all rats, as previously described (19). This procedure allowed for the phase of the estrus cycle to be determined by daily analysis of the types of cells that sloughed off the vaginal epithelium. With this approach, four different stages can be observed, as follows: Proestrus (nucleated epithelial cells), estrus (cornified cells), metestrus (some cornified cells in addition to nucleated cells and a large number of leukocytes) and diestrus (leukocyte infiltration). Collected vaginal fluid was placed on glass slides and examined by light microscopy. In the Sham group, estrous cycle regularity was confirmed by the presence of vaginal epithelial cells characteristic of each of the four aforementioned stages. In the remaining groups, the absence of the estrous cycle was confirmed by a permanent diestrus phase.

Hormone assays. The level of 17 $\beta$-Estradiol (E2) in serum was assessed using an enzyme-linked immunosorbent assay kit (cat. no. 10006315; MultiSciences Biotech Co., Ltd., Hangzhou, China) according to manufacturer's protocol. Absorbance was measured at $450 \mathrm{~nm}$ within $15 \mathrm{~min}$ (Multiskan FC; Thermo Fisher Scientific, Inc., Waltham, MA, USA).

Measurement of ROS, malondialdehyde (MDA), glutathione peroxidase (GSH-px), superoxide dismutase (SOD) and catalase (CAT) activities. The rats were anaesthetized with $10 \%$ chloral hydrate, and sacrificed by decapitation after 13 weeks. A $1-2 \mathrm{~cm}$ skin incision was first made, $0.5 \mathrm{~cm}$ below the rib and $1 \mathrm{~cm}$ next to the spine. Then the subcutaneous tissue, muscle and peritoneum were cut in turn. Following the opening of the peritoneal cavity, the rat kidney was identified and a white cellulite beneath it, exposing the soybean-size glandular spheres (the ovaries) which were removed by wire. Finally, following the confirmation that there was no intra-abdominal peritoneal bleeding, the peritoneum and skin were closed layer by layer, and the operating area cleaned. Following the surgery, in a warm environment, the rats were released into cages on wakening and given intraperitoneal injections of penicillin 160,000 units/rat three days after the surgery to prevent infection. The myocardium (100 mg) was homogenized in cold saline. The homogenate was then centrifuged at $900 \mathrm{x} g$ for $15 \mathrm{~min}$. The activities of ROS (cat. no. E004), MDA (cat. no. A003-2), GSH-px (cat. no. A005), SOD (cat. no. A001-1) and CAT (cat. no. A007-2; all from Nanjing Jiancheng Biological Engineering Institute, Nanjing, China) were determined using these assay kits according to the manufacturer's protocols.

Hematoxylin and eosin (H\&E) staining of myocardium. Myocardium samples were fixed in $4 \%$ paraformaldehyde for $24 \mathrm{~h}$ at room temperature and stained with H\&E, according to standard techniques. Briefly, the samples were embedded in paraffin and sections $(5-\mu \mathrm{m})$ were obtained. The samples were then dewaxed with xylene, rehydrated through an alcohol gradient and were stained with H\&E for light microscopy. Images were captured using a light microscope linked to a digital charge-coupled device camera (Olympus Corporation, Tokyo, Japan).

Protein extraction and western blotting. Total cellular proteins were extracted from heart tissues using Radioimmunoprecipitation Lysis Buffer (Beyotime Institute of 
Biotechnology, Shanghai, China). Protein concentration was measured using a Bicinchoninic Acid Protein Assay kit (Beijing Dingguo Changsheng Biotechnology Co. Ltd., Beijing, China). Western blotting was conducted to assess the protein expression levels of B cell lymphoma-2 ( $\mathrm{Bcl} 2)$, Bcl2-associated X protein (Bax), cleaved caspase-9, cleaved caspase-3 and phosphorylated (p)-protein kinase B (Akt). Briefly, $40 \mu \mathrm{g}$ protein samples were separated by $10 \%$ SDS-PAGE, after which the proteins were transferred onto polyvinylidene fluoride membranes (EMD Millipore, Billerica, MA, USA). The membranes were then incubated overnight $\left(4^{\circ} \mathrm{C}\right)$ with antibodies against $\beta$-actin (cat. no. Sc-130300; Santa Cruz Biotechnology, Inc., Dallas, TX, USA), Bax (cat. no. Sc-4239; Santa Cruz Biotechnology, Inc.), Bcl2 (cat. no. Sc-509; Santa Cruz Biotechnology, Inc.), caspase-9/cleaved caspase-9 (cat. no. 9504; Cell Signaling Technology, Inc., Danvers, MA, USA), caspase-3/cleaved caspase-3 (cat. no. 9509; Cell Signaling Technology, Inc.), Akt (cat. no. 9662, Cell Signaling Technology, Inc.) and p-Akt (cat. no. 9667, Cell Signaling Technology, Inc.). The dilution of $\beta$-actin, Bax and $\mathrm{Bcl} 2$ antibodies was 1:500, and for caspase-9/cleaved caspase-9, caspase-3/cleaved caspase-3, Akt and p-Akt antibodies 1:1,000. The membranes were then incubated with a horseradish peroxidase-conjugated goat anti-rabbit secondary antibody (cat. no. Sc-2004; 1:3,000; Santa Cruz Biotechnology, Inc.) for $1 \mathrm{~h}$ at room temperature. Protein bands were visualized using an enhanced chemiluminescence kit (Thermo Fisher Scientific, Inc.). Protein expression was normalized to $\beta$-actin. ImageJ software version 1.45 (National Institutes of Health, Bethesda, MD, USA) was used to perform densitometric analysis.

Statistical analysis. All experiments were carried out at least in duplicate. Data are presented as the mean \pm standard deviation. Statistical analysis was performed with one-way analysis of variance followed by Kruskal Wallis test using the GraphPad Prism 5 software package (GraphPad, La Jolla, CA, USA). $\mathrm{P}<0.05$ was considered to indicate a statistically significant difference.

\section{Results}

$L B P$ increases the serum levels of E2 and modifies the OVX-induced alterations in cardiac tissue. In order to confirm that OVX was successful, serum E2 levels were measured. A significant reduction in serum E2 levels was observed in the OVX group compared with in the Sham group $(\mathrm{P}<0.01)$, thus indicating that OVX was successful. Treatment with high-dose LBP and estradiol valerate increased serum E2 levels compared with in OVX rats $(\mathrm{P}<0.01)$. However, there was no significant difference in E2 levels in the serum between the LBP-L and OVX groups (Fig. 1A). In the Sham group, myocardial fibers were arranged regularly with clear striations, without any damage or necrosis in the tissue (Fig. 1B). Histopathological sections of the OVX group displayed disorganized fibers and increased dilatation of intercellular spaces. Conversely, high-dose LBP and estradiol valerate modified the OVX-induced alterations in cardiac tissue (Fig. 1C-F).

$L B P$ recovers the antioxidant status in OVX rats. As illustrated in Fig. 2, various parameters associated with oxidative stress were detected. Enhanced ROS and MDA activity was detected in the myocardium of the OVX group $(\mathrm{P}<0.01)$. Administration of a high-dose of LBP exerted a significant protective effect on OVX rats $(\mathrm{P}<0.01)$. In addition, administration of estradiol valerate exerted a significant protective effect on MDA only in OVX rats $(\mathrm{P}<0.01)$. GSH-px CAT and SOD are markers of the antioxidant defense system. As presented in Fig. 2, OVX resulted in a decrease in GSH-px and CAT activity $(\mathrm{P}<0.01)$, whereas in the LBP-H and Est groups GSH-px and CAT were significantly increased in the myocardium compared with in OVX rats $(\mathrm{P}<0.05)$. No alterations in SOD were detected among the various groups. These findings indicated that administration of LBP may recover the antioxidant status in OVX rats.

LBP alleviates apoptosis in OVX rats. Bax, as a proapoptotic protein, $\mathrm{Bcl} 2$, as an anti-apoptotic protein, and caspase- 9 and caspase-3 are important indicators of apoptosis. As demonstrated in Fig. 3, OVX resulted in an increase in Bax, cleaved caspase- 9 and cleaved caspase- 3 expression in the myocardium $(\mathrm{P}<0.01)$. Administration of high-dose LBP and estradiol valerate exerted a significant protective effect on OVX rats, as evidenced by the decreased expression of apoptotic proteins $(\mathrm{P}<0.05)$. In addition, OVX resulted in a decrease in $\mathrm{Bcl} 2$ expression in the myocardium $(\mathrm{P}<0.01)$. However, $\mathrm{Bcl} 2$ expression was enhanced in the myocardium of the LBP-H and Est groups compared with in OVX rats $(\mathrm{P}<0.01)$.

LBP alleviates apoptosis via the Akt signaling pathway in OVX rats. The Akt signaling pathway is involved in apoptosis. As illustrated in Fig. 4, OVX resulted in a decrease in the phosphorylation of Akt in the myocardium $(\mathrm{P}<0.01)$. Administration of high-dose LBP resulted in a significant increase in the expression of p-Akt $(\mathrm{P}<0.01)$.

\section{Discussion}

The present study demonstrated that LBP can improve antioxidant status, ameliorate oxidative stress-induced cell apoptosis and increase phosphorylation of Akt in the myocardium of OVX rats. OVX-induced cardiac injury suggests that estrogens may be associated with the maintenance of normal cardiac function. This relationship has already been suggested by other studies $(20,21)$. Estrogen has also been reported to provide cardiac protection in various models of cardiac disease $(22,23)$.

Oxidative stress represents an imbalance between the production and manifestation of ROS and their detoxification. Oxidative stress may serve an important pathophysiological role during the menopause, and is considered one of the main causative factors of various cardiovascular disorders, including postmenopausal cardiovascular disorders $(24,25)$. A number of studies have demonstrated that OVX may result in a decrease in antioxidative biomarkers and an increase in MDA content (oxidative biomarker) in the myocardium $(26,27)$. SOD, CAT and GSH comprise the antioxidant defense system; SOD and CAT are important components of this system. SOD is considered the most important antioxidant enzyme that provides defense against oxidative stress, particularly oxygen radicals. SOD scavenges superoxide by converting it to peroxide, which 

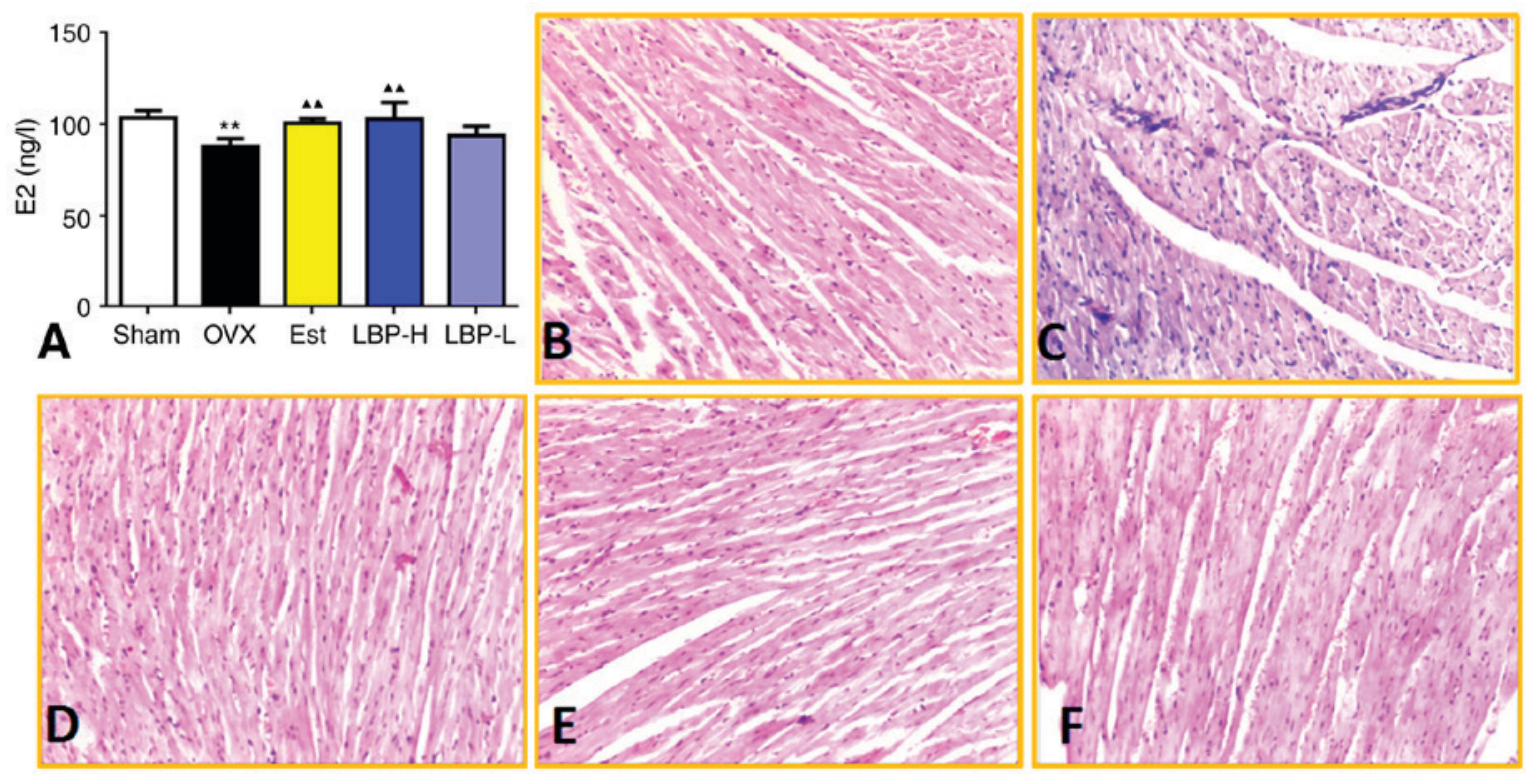

Figure 1. Effects of LBP on serum E2 levels and histopathological examination of cardiac tissue. (A) Effects of LBP therapy on serum E2 levels. A significant reduction in serum E2 levels was observed in OVX rats, whereas E2 levels were increased in the LBP-H group compared with in the OVX group (n=6/group). ${ }^{* *} \mathrm{P}<0.01$ vs. the Sham group; ${ }^{\boldsymbol{\Delta}} \mathrm{P}<0.01$ vs. the OVX group. (B-F) Representative photomicrographs of cardiac tissue from the Sham, OVX, Est, LBP-H and LBP-L groups, respectively. Histopathological sections of the OVX group displayed disorganized fibers and increased dilatation of intercellular spaces, whereas these alterations were modified in the LBP-H and Est groups. Hematoxylin and eosin staining; magnification, x20. E2, 17/-estradiol; Est, estradiol valerate group; LBP, Lycium barbarum polysaccharides; LBP-H, high-dose LBP group; LBP-L, low-dose LBP group; OVX, ovariectomy group.

A

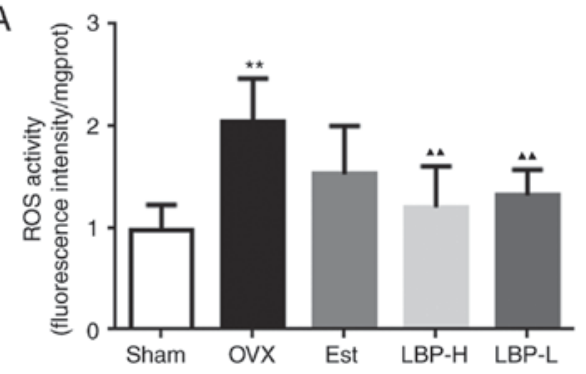

C

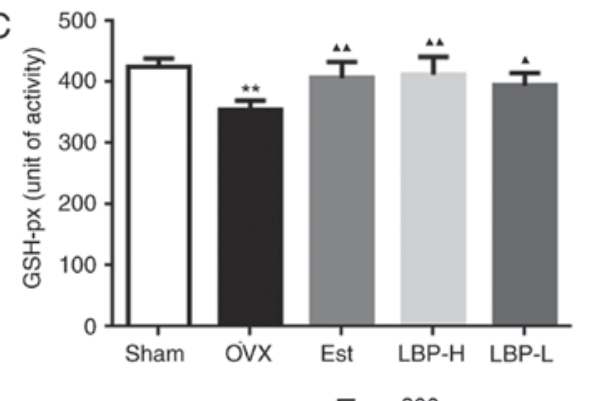

B

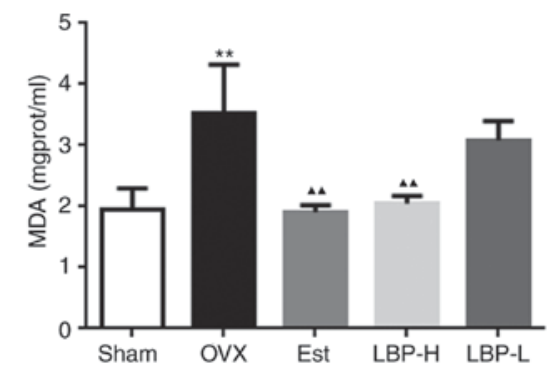

D

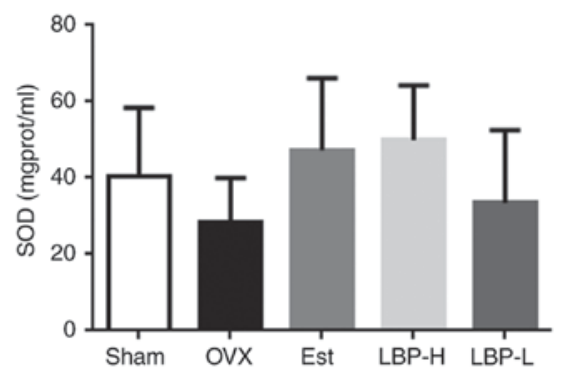

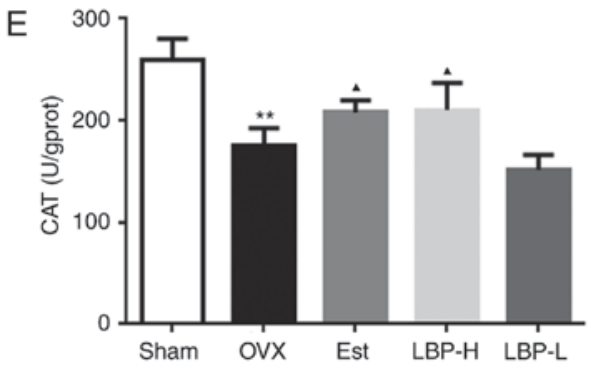

Figure 2. Effects of LBP and OVX on oxidative status in the myocardium of rats. (A and B) Effects of LBP on ROS and MDA activity. A significant increase in ROS and MDA was detected in the myocardium of OVX rats. Conversely, in the LBP-H group ROS and MDA activity was significantly decreased compared with in the OVX group. (C-E) Effects of LBP on GSH-px, SOD and CAT activity. A significant decrease in GSH and CAT was detected in the myocardium of OVX rats. Conversely, in the LBP-H group GSH and CAT activity was significantly increased compared with in the OVX group ( $\mathrm{n}=6 /$ group). ${ }^{* *} \mathrm{P}<0.01 \mathrm{vs}$. the Sham group; ${ }^{\mathbf{A}} \mathrm{P}<0.05,{ }^{\mathbf{\Delta}} \mathrm{P}<0.01$ vs. the OVX group. CAT, catalase; Est, estradiol valerate group; GSH-px, glutathione peroxidase; LBP, Lycium barbarum polysaccharides; LBP-H, high-dose LBP group; LBP-L, low-dose LBP group; MDA, malondialdehyde; OVX, ovariectomy group; ROS, reactive oxygen species; SOD, superoxide dismutase. 

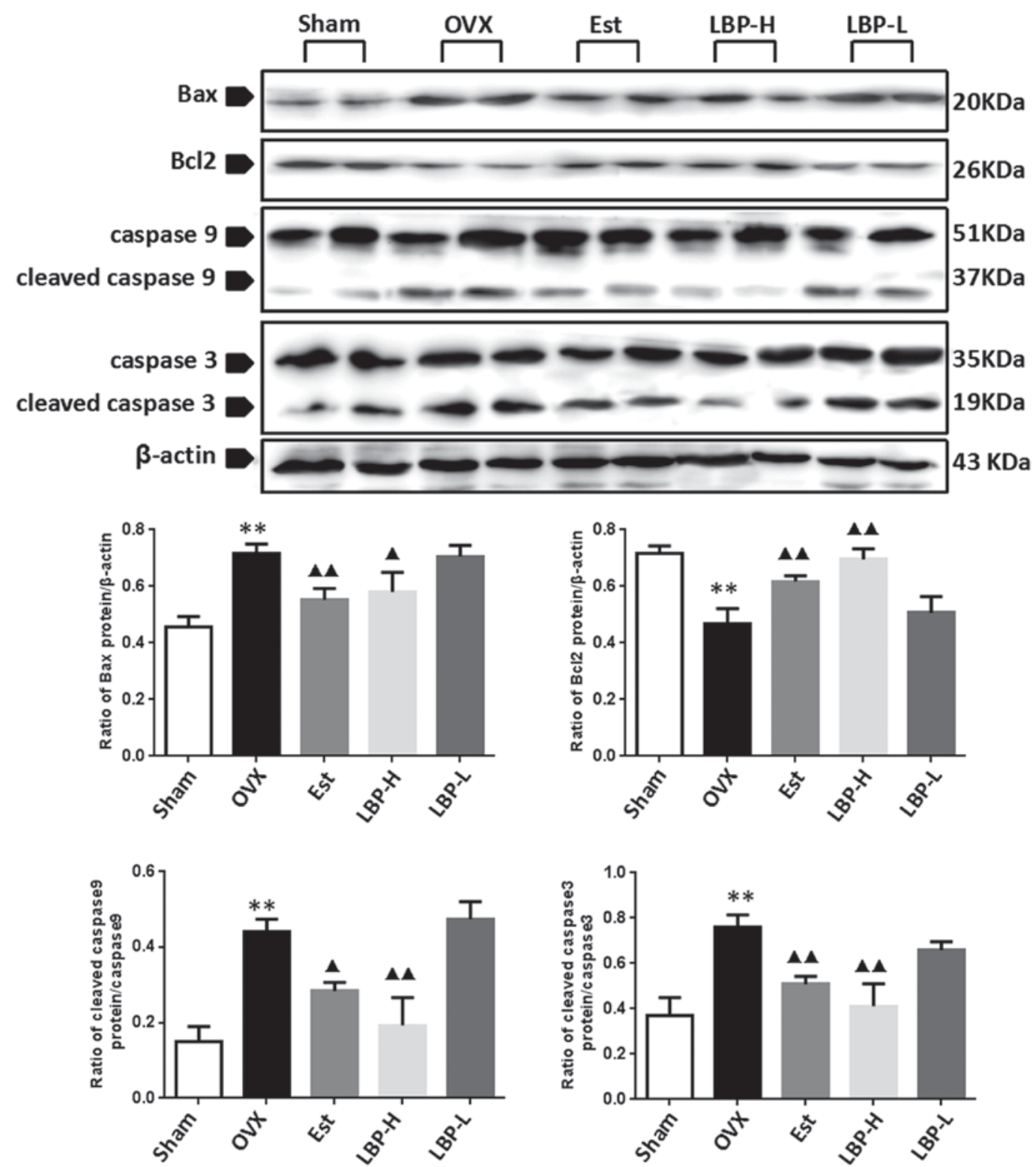

Figure 3. Effects of LBP and OVX on apoptotic protein expression in the myocardium. The expression levels of apoptotic proteins were determined by western blotting. A significant increase in Bax, cleaved caspase- 9 and cleaved caspase-3, and a decrease in Bcl2 expression were detected in the myocardium of OVX rats. In the LBP-H group, the expression levels of Bax, cleaved caspase- 9 and cleaved caspase- 3 were decreased, and Bcl 2 expression was increased compared with in the OVX group ( $\mathrm{n}=3$ /group). ${ }^{* *} \mathrm{P}<0.01$ vs. the Sham group; ${ }^{\Delta} \mathrm{P}<0.05,{ }^{\boldsymbol{\Delta}} \mathrm{P}<0.01$ vs. the OVX group. Bax, Bcl2-associated $\mathrm{X}$ protein; Bcl2, $\mathrm{B}$ cell lymphoma-2; Est, estradiol valerate group; LBP, Lycium barbarum polysaccharides; LBP-H, high-dose LBP group; LBP-L, low-dose LBP group; OVX, ovariectomy group.

in turn is destroyed by CAT. Therefore, SOD and CAT act in a mutually supportive way with antioxidant enzymes to provide a protective defense against ROS. GSH-px catalyzes the reductive action of GSH to $\mathrm{H}_{2} \mathrm{O}_{2}$, in order to protect the integrity and functions of the plasma membrane (28-30). Ji et al (31) demonstrated that estrogens are able to suppress the overproduction of ROS. Numerous studies (32-34) have revealed the protective effects of E2 on GSH synthesis. Therefore, OVX may significantly alter the stimulatory effects of E2 on GSH synthesis.

In the present study, a significant reduction in serum E2 levels was observed in OVX rats. Conversely, 12-week treatment with $250 \mathrm{mg} / \mathrm{kg} \mathrm{LBP}$, which has estrogen-like effects, following OVX improved serum E2 levels. Furthermore, OVX induced an imbalance in oxidative stress status: ROS and MDA activity was increased, whereas GSH-px and CAT activity was decreased in cardiac tissues. LBP treatment decreased ROS and MDA activity, and increased GSH-px and CAT activity in cardiac tissues. These results indicated that an important association exists between oxidative stress and LBP-induced cardioprotective effects.

Lycium barbarum $\mathrm{L}$ is a well-known antioxidant traditional Chinese medicine. The aqueous extract of Lycium barbarum $\mathrm{L}$ has been reported to exert marked antioxidant activity in vivo and in vitro $(35,36)$. LBP is considered the main active component in Lycium barbarum $\mathrm{L}$, which possesses numerous bioactivities, including anti-aging, anticancer, immunomodulatory and antioxidative effects (37). Li demonstrated that LBP $(20-50 \mathrm{mg} / \mathrm{kg})$ protects liver and kidney tissue from oxidative damage in streptozotocin-induced diabetic rats (38). 


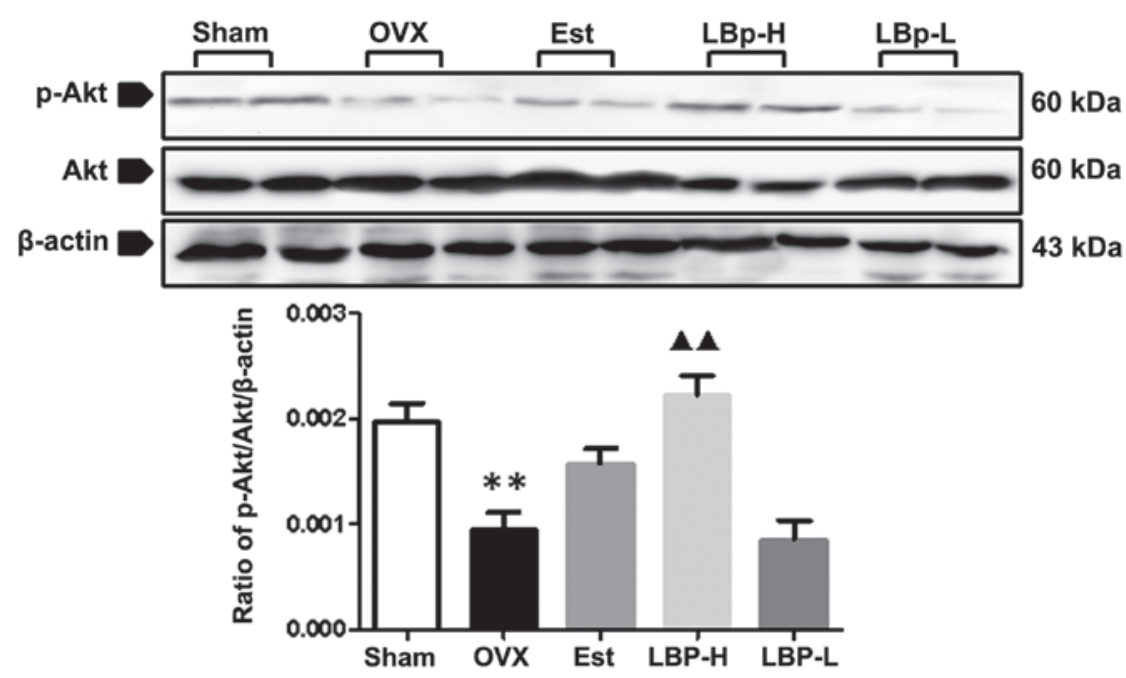

Figure 4. Effects of LBP and OVX on phosphorylation of Akt in the myocardium. The levels of p-Akt were determined by western blotting. A significant decrease in p-Akt protein expression was detected in the OVX rats. However, in the LBP-H group, the protein expression levels of p-Akt were significantly increased compared with in the OVX group ( $\mathrm{n}=3$ /group). ${ }^{* *} \mathrm{P}<0.01$ vs. the Sham group; ${ }^{\boldsymbol{\Delta}} \mathrm{P}<0.01$ vs. the OVX group. Akt, protein kinase $\mathrm{B}$; Est, estradiol valerate group; LBP, Lycium barbarum polysaccharides; LBP-H, high-dose LBP group; LBP-L, low-dose LBP group; OVX, ovariectomy group; p-Akt, phosphorylated-Akt.

Furthermore, Luo et al (39) indicated that LBP could alleviate heat-induced damage of rat testes, and $\mathrm{H}_{2} \mathrm{O}_{2}$-induced DNA damage in mouse testicular cells, by increasing their resistance to oxidative stress-induced injury. The results of the present study demonstrated that in rats treated with LBP $(250 \mathrm{mg} / \mathrm{kg}), \mathrm{OVX}$-induced oxidative injury in cardiac tissue was less apparent compared with in the OVX group; LBP was able to decrease ROS and MDA activity, thus improving OVX-induced abnormalities. In addition, LBP significantly $(\mathrm{P}<0.05)$ increased CAT and GSH-px activity in the heart tissues of OVX rats. Taken together, LBP exerted indirect effects to alleviate OVX-induced cardiomyocyte damage.

Oxidative stress can activate cell apoptosis signaling, resulting in the induction of apoptosis in various cell types (40). Bax, as a proapoptotic protein, and $\mathrm{Bcl} 2$, as an anti-apoptotic protein, and caspase- 9 and caspase-3, are important indicators of apoptosis. In the OVX group, increased $\mathrm{Bax}$ and decreased $\mathrm{Bcl} 2$ protein expression was detected in the cardiac muscle, thus indicating that the cardiomyoctes in these rats were undergoing apoptosis. In addition, there were significant differences in Bax and $\mathrm{Bcl} 2$ protein expression between the OVX and LBP-H groups. These results suggested that the balance between anti-apoptotic and proapoptotic factors was disrupted by OVX. Compared with the OVX group, cardiomyocyte apoptosis was alleviated by LBP $(250 \mathrm{mg} / \mathrm{kg})$. In addition to Bcl 2 and Bax, caspase-3 and caspase-9 deactivation also contributed to LBP-mediated cardiac protection. These findings indicated that estrogen and LBP may serve a similar role in regulating cardiomyocyte function by inhibiting apoptosis.

Previous studies have reported that overproduction of ROS is associated with three pathways: Extracellular auto-oxidation, intracellular metabolism by monoamine oxidase and direct inhibition of the mitochondrial respiratory chain $(41,42)$. Furthermore, the generation of intracellular ROS suppresses Akt phosphorylation, which induces activation of caspase-9 and caspase-3, which finally leads to cell apoptosis (42). In the present study, in the OVX group, cleaved caspase- 3 and caspase-9 protein expression was increased, indicating the occurrence of cell apoptosis, whereas in the LBP group, cleaved caspase-3 and caspase-9 protein expression was decreased in cardiac tissues. In addition, the phosphorylation of Akt was decreased in the cardiac tissues of the OVX group; however, following treatment with a high dose of LBP, the phosphorylation of Akt was improved.

In conclusion, LBP may increase activity levels of the antioxidative markers CAT and GSH-px, and decrease activity levels of the oxidative markers MDA and ROS in the myocardium of OVX rats. Therefore, LBP may ameliorate oxidative stress-induced cardiac damage in OVX rats. LBP is associated with cardiac protection by inhibiting the apoptotic signaling pathway in response to oxidative stress; this finding is associated with the Akt signaling pathway in the myocardium.

\section{Acknowledgements}

Not applicable.

\section{Funding}

The present study was supported by the Liaoning University of Traditional Chinese Medicine Key Laboratory of Ministry of Education for TCM Viscera-State Theory and Applications open fund (grant no. F14-231-1-17), the Liaoning University of Traditional Chinese Medicine College Students' Innovative Entrepreneurial Training Plan (grant no. 201510162000002), and The 60th China Postdoctoral Science Fundation Grant (grant no. 2016M601331).

\section{Availability of data and materials}

The datasets used and/or analyzed during the current study are available from the corresponding author on reasonable request. 


\section{Authors' contributions}

NY performed the animal breeding, the detection of experimental indicators and the statistical analysis. NS conducted the detection of experimental indicators. CL was mainly responsible for the statistical analysis. GY designed the study. All authors have read and approved the manuscript.

\section{Ethics approval and consent to participate}

The present study was approved by and followed the guidelines of the Animal Ethics Committee of Liaoning University of Traditional Chinese Medicine (Shenyang, China; permit no. 2011-167).

\section{Patient consent for publication}

Not applicable.

\section{Competing interests}

The authors declare that they have no competing interests.

\section{References}

1. Mendelsohn ME and Karas RH: Molecular and cellular basis of cardiovascular gender differences. Science 308: 1583-1587, 2005.

2. Rossouw JE, Prentice RL, Manson JE, Wu L, Barad D Barnabei VM, Ko M, LaCroix AZ, Margolis KL and Stefanick ML: Postmenopausal hormone therapy and risk of cardiovascular disease by age and years since menopause. JAMA 297: 1465-1477, 2007.

3. Arias-Loza PA, Muehlfelder M and Pelzer T: Estrogen and estrogen receptors in cardiovascular oxidative stress. Pflugers Arch 465: 739-746, 2013.

4. Wang F, Xiao J, Shen Y, Yao F and Chen Y: Estrogen protects cardiomyocytes against lipopolysaccharide by inhibiting autophagy. Mol Med Rep 10: 1509-1512, 2014.

5. Rossouv JE, Prentice RE, Manson JE, Wu L, Barad D, Barnabei VM, Ko M, LaCroix AZ, Margolis KL and Stefanick ML: Postmenopausal hormone 1therapy and risk of cardiovascular disease by age and years since menopause. JAMA 297: 1465-1477, 2007.

6. Babiker FA, DeWindt LJ, Van Eickels M, Grohe C, Meyer R and Doevendans PA: Estrogenic hormone action in the heart: Regulatory network and function. Cardiovasc Res 53: 709-719, 2002.

7. Zegura B, Keber I, Sebestjen M and Koenig W: Double blind, randomized study of estradiol replacement therapy on markers of inflammation, coagulation and fibrinolysis. Atherosclerosis 168 : 123-129, 2003.

8. Miller VM and Duckles SP: Vascular actions of estrogens: Functional implications. Pharmacol Rev 60: 210-241, 2008.

9. Knowlton AA and Lee AR: Estrogen and the cardiovascular system. Pharmacol Ther 135: 54-70, 2012.

10. Ni W, Gao T and Wang H: Anti-fatigue activity of polysaccharides from the fruits of four Tibetan plateau indigenous medicinal plants. J Ethnopharmacol 150: 529-535, 2013.

11. Xiao J, Xing F, Huo J, Fung ML, Liong EC, Ching YP, Xu A, Chang RC, So KF and Tipoe GL: Lycium barbarum polysaccharides therapeutically improve hepatic functions in non-alcoholic steatohepatitis rats and cellular steatosis model. Sci Rep 4: 5587, 2014.

12. Giordano FJ: Oxygen, oxidative stress, hypoxia and heart failure. J Clin Invest 115: 500-508, 2005.

13. Saglam H, Kaya E, Cemek M, Ciçek Y, Kulac M and Karaca S: No apparent correlation between Behçet's disease and oxidative stress disturbance. Clin Hemorheol Microcric 44: 287-296, 2010

14. El-Sayyad HI, AI-Haggar MS, EI-Ghawet HA and Bakr IH: Cardiomyopathy and angiogenesis defects of Wistar rat fetuses of diabetic and hypercholesterolemic mothers. Nutrition 28: e33-e43, 2012.
15. Tang Q, Len Q, Liu Z and Wang W: Overexpression of miR-22 attenuates oxidative stress injury in diabetic cardiomyopathy via Sirt 1. Cardiovasc Ther 36, 2017 doi: 10.1111/1755-5922.12318.

16. Mallat Z, Philip I, Lebret M, Chatel D, Maclouf J and Tedgui A: Elevated levels of 8-iso-prostaglandin F2alpha in pericardial fluid of patients with heart failure: A potential role for in vivo oxidant stress in ventricular dilatation and progression to heart failure. Circulation 97:1536-1539, 1998.

17. Xin YF, Wan LL and Peng JL: Alleviation of the acute doxorubicin-induced cardiotoxicity by Lycium barbarum polysaccharides through the suppression of oxidative stress. Food Chem Toxicol 49: 259-264, 2011.

18. Lu SP and Zhao PT: Chemical characterization of Lycium barbarum polysaccharides and their reducing myocardial injury in ischemia/reperfusion of rat heart. Int J Biol Macromol 47: 681-684, 2010.

19. Poumeau-Delille G: Techniques biologiques en endocrinologie experimentale chez rat. Masson and Cie, 1953.

20. Bolego C, Cignarella A, Ruzza R, Zaarour C, Messi E, Zanisi M and Puglisi L: Differential effects of low- and high-dose estrogen treatments onvascular responses in female rats. Life Sci 60: 2291-2302, 1997.

21. Persky AM, Green PS, Stubley L, Howell CO, Zaulyanov L, Brazeau GA and SimpkinsJW:Protectiveeffect of estrogens against oxidativedamage to heart and skeletal muscle in vivo and in vitro. Proc Soc Exp Biol Med 223: 59-66, 2000.

22. Patten RD, Pourati I, Aronovitz MJ, Ali AA, Eder S, Force T, Mendelsohn ME and Karas RH: 17 Beta-estradiol differentially affects left ventricular and cardiomyocyte hypertrophy following myocardial infarction and pressure overload. J Cardiac Fail 14: 245-253, 2008

23. TagashiraH,Bhuiyan S, ShiodaNandFukunagaK:Distinctcardioprotective effects of $17 \beta$-estradiol and dehydroepiandrosterone on pressure overload-induced hypertrophy in ovariectomized female rats. Menopause 12: 1317-1326, 2011.

24. Aldini G, Yeum KJ, Niki E, et al: Biomarkers for antioxidant defense and oxidative damage: Principles and practical applications. Ames. Wiley-Blackwell. 2010.

25. Castelao JE and Gago-Dominguez M: Risk factors for cardiovascular disease in women: Relationship to lipid peroxidation and oxidative stress. Med Hypotheses 71: 39-44, 2008.

26. ZhuX,TangZ,Cong B,DuJ,WangC,WangL,NiX andLuJ:Estrogens increase cystathionine- $\gamma$-lyase expression and decrease inflammation and oxidative stress in the myocardium of ovariectomized rats. Menopause 20: 1084-1091, 2013.

27. Tang Z, Wang Y, Zhu X, Ni X and Lu J: Exercise increases cystathionine- $\gamma$-lyase expression and decreases the status of oxidative stress in myocardium of ovariectomized rats. Int Heart J 57: 96-103, 2016.

28. Liochev SI and Fridovich I: Arch Biochem Biophys 337: 115-120, 1997.

29. Wu HT, He XJ, Hong YK, Ma T, Xu YP and Li HH: Chemical characterization of lycium barbarum polysaccharides and its inhibition against liver oxidative injury of high-fat mice. Int J Biol Macromol 46: 540-543, 2010.

30. Aksakal E, Akaras N, Tanboga IH, Kurt M, Halici Z, Odabasoglu F and Unal B: Relationship between oxidative stress and cardiomyopathic changes in ovariectomized rats. Cardiology 119: 235-241, 2011.

31. Ji H, Zheng W, Menini S, Pesce C, Kim J, Wu X, Mulroney SE and Sandberg K: Female protection in progressive renal disease is associated with estradiol attenuation of superoxide production. Gend Med 4: 56-71, 2007.

32. Urata Y, Ihara Y, Murata H, Goto S, Koji T, Yodoi J, Inoue S and Kondo T: 17Beta-estradiolprotectsagainstoxidativestress-induced cell death through the glutathione/glutaredoxin-dependent redox regulation of Akt in myocardiac H9c2 cells. J Biol Chem 281: 13092-13102, 2006.

33. Sawicka E and Długosz A: The role of $17 \beta$-estradiol metabolites in chromium-induced oxidative stress. Adv Clin Exp Med 26: 215-221, 2017.

34. Baeza I, Fdez-Tresguerres J, Ariznavarreta C and De la Fuente M: Effects of growth hormone, melatonin, oestrogens and phytoestrogens on the oxidized glutathione (GSSG)/reduced glutathione $(\mathrm{GSH})$ ratio and lipid peroxidation in aged ovariectomized rats. Biogerontology 11: 687-701, 2010.

35. Wu SJ, Ng LT and Lin CC: Antioxidant activities of some common ingredients of traditional Chinese medicine, Angelica sinensis, Lycium barbarum and Poriacocos. Phytother Res 18: 1008-1012, 2004. 
36. Luo Q, Cai Y, Yan J, Sun M and Corke H: Hypoglycemic and hypolipidemic effects and antioxidant activity of fruit extracts from Lycium barbarum. Life Sci 76: 137-149, 2004.

37. Potterat O: Goji (Lycium barbarum and L. Chinense): Phytochemistry, pharmacology and safety in the perspective of traditional uses and recent popularity. Planta. Med 76: 7-19, 2010.

38. Li XM: Protective effect of Lycium barbarum polysaccharides on streptozotocin-induced oxidative stress in rats. Int J Biol Macromol 40: 461-465, 2007.

39. Luo Q, Li Z, Huang X, Sun M and Corke H: Lycium barbarum polysaccharides: Protective effects against heat-induced damage of rat testes and $\mathrm{H}_{2} \mathrm{O}_{2}$-induced DNA damage in mouse testicular cells and beneficial effect on sexual behavior and reproductive function of hemicastrated rats. Life Sci 79: 613-621, 2006.
40. Martindale JL and Holbrook N: Cellular response to oxidative stress: Signaling for suicide and survival. J Cell Physiol 192: $1-15,2002$.

41. Saito Y, Nishio K, Ogawa Y, Kinumi T, Yoshida Y, Masuo Y and Niki E: Molecular mechanisms of 6-hydroxydopamine-induced cytotoxicity in PC12 cells: Involvement of hydrogen peroxide-dependent and -independent action. Free Radic Biol Med 42: 675-685, 2007.

42. Fujita H, Ogino T, Kobuchi H, Fujiwara T, Yano H, Akiyama J, Utsumi K and Sasaki J: Cell-permeable cAMP analog suppresses 6-hydroxydopamine-induced apoptosis in PC12 cells through the activation of the Akt pathway. Brain Res 1113: $10-23,2006$. 